\title{
SYNTHESIS OF 1-(3,3,3-TRIFLUORO-1-PROPENYLSULFONYL)PYRROLIDINE AND ITS MICHAEL ADDITION WITH SOME SELECTED NUCLEOPHILES
}

\author{
Hiroyasu Tsuge, Kensuke Takumi, Takashi Okano and Shoji Eguchi" \\ Department of Molecular Design and Engineering, Graduate School of Engineering, \\ Nagoya University, Furo-cho, Chikusa-ku,. Nagoya 464-01, Japan
}

\begin{abstract}
Trifluoro-I-propenylsulfonyl)pyrrolidine $\underline{1}$ was synthesized from $N$-mesylpyrrolidine and ethyl trifluoroacetate. Michael addition of $\underline{1}$ with some selected nucleophiles including enolate anions and an amine gave the adducts regioselectively in high yields (58-100\%), although no adducts with organometallics were obtained.
\end{abstract}

Much attention has been addressed to trifluoromethylated compounds because they often exhibit unique biological activities (1). For introduction of the trifluoromethyl group into a carbon skeleton, the use of a proper building block, which already has the trifluoromethyl group in it, is one of the most efficient approaches (2). From this point of view, we are interested in the use of trifluoropropenylsulfonyl compounds for the synthesis of trifluoromethylated compounds (3). Some organic compounds containing the trifluoropropenyl group also exhibit remarkable pharmaceutical activities (4). In this paper, we wish to report the synthesis of 1-(3,3,3trifluoro-1-propenylsulfonyl)pyrrolidine $\underline{1}$ as a new class of trifluoromethylated building blocks and the Michael addition of 1 with some selected nucleophiles.

Michael additions have been employed as powerful carbon - carbon bond or carbon - heteroatom bond formation methodologies for organic synthesis (5). A number of examples of Michael additions using the olefin with an electron-withdrawing group were reported (5), however, there seems to be no report of the use of $\alpha, \beta$ unsaturated sulfonamide as a Michael acceptor.

As the starting amine, we chose the five-membered pyrrolidine because this Michael addition could be extended into the asymmetric reaction by use of optically active pyrrolidine derivatives. The sulfonamide $\underline{1}$ was synthesized as outlined in Scheme 1. Mesylation of pyrrolidine with methanesulfonyl chloride and triethylamine at $0{ }^{\circ} \mathrm{C}$ gave the amide $\underline{2}$ ( $95 \%$ yield). Treatment of $\underline{2}$ with $n$-BuLi at $-78{ }^{\circ} \mathrm{C}-0{ }^{\circ} \mathrm{C}$ followed by addition of ethyl trifluoroacetate afforded the ketone $\underline{3} . \mathrm{NaBH}_{4}$ reduction of $\underline{3}$ to the alcohol $\underline{4}$ and the following dehydration with 


\section{Scheme 1}
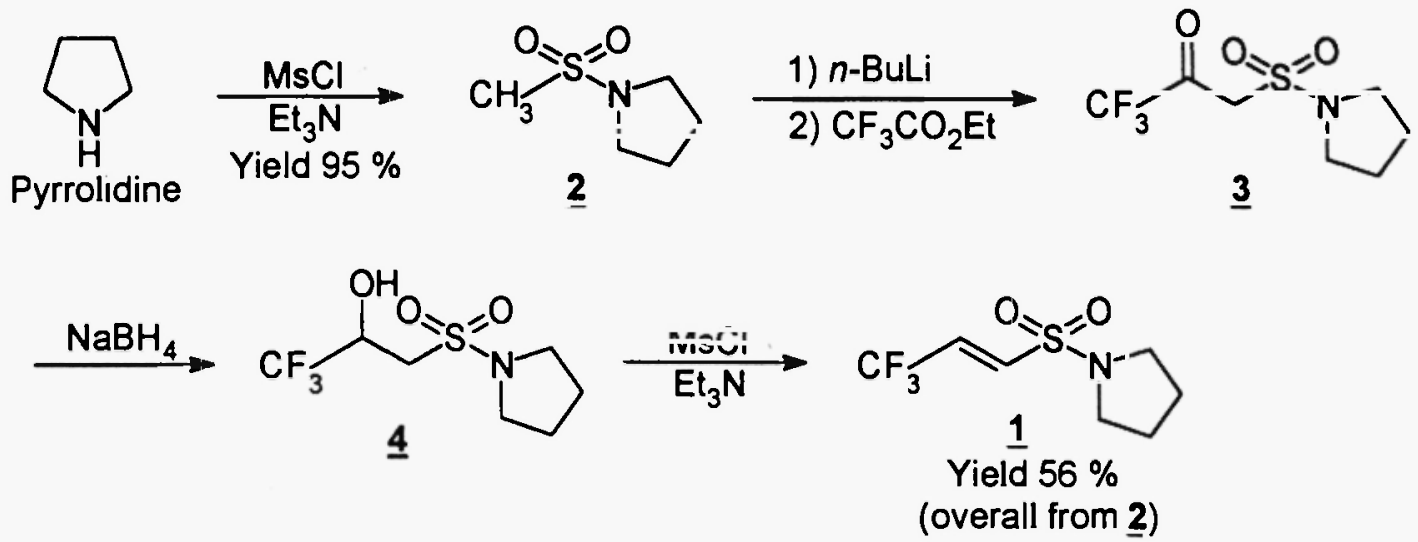

methanesulfonyl chloride in the presence of an excess amount of triethylamine to give the sulfonamide 1 in good yield ( $56 \%$ yield from 2 ).

Michael addition of $\underline{1}$ with some selected nucleophiles was examined as summarized in Table 1. Michael addition of $\underline{1}$ with dimethyl malonate deprotonated with $\mathrm{NaH}$ at room temperature gave the adduct $\underline{\mathbf{5}}$ quantitatively. When lithium enolate of acetophenone was used as a nucleophile, the Michael addition took place at $-78{ }^{\circ} \mathrm{C}$ to afford the adduct 6 in $66 \%$ yield. Since the Michael addition of sodium benzylamide was very slow at room temperature, the reaction was carried out at $80^{\circ} \mathrm{C}$ for $3 \mathrm{~h}$ to obtain the adduct $\underline{7}$ in $58 \%$ yield. Several attempts of the reaction of 1 with organometalic reagents (alkyl lithiums, Grignard reagents, and organo copper reagents) failed probably because of the relatively high acidity of the hydrogen adjacent to the sulfonyl group in 1 .

Table 1. Michael addition of 1 with some selected nucleophiles

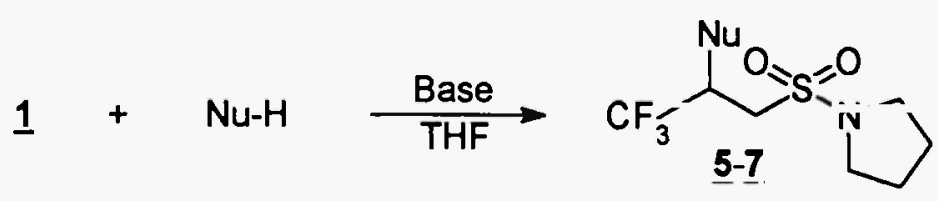

\begin{tabular}{ccccc}
$\mathrm{Nu}-\mathrm{H}$ & Base & Reaction conditions & Product & Yield (\%) \\
\hline $\mathrm{CH}_{2}\left(\mathrm{CO}_{2} \mathrm{CH}_{3}\right)_{2}$ & $\mathrm{NaH}$ & $\mathrm{rt}, 1 \mathrm{~h}$ & $\underline{\underline{5}}$ & 100 \\
$\mathrm{CH}_{3} \mathrm{COPh}$ & $\mathrm{LDA}$ & $-78^{\circ} \mathrm{C}, 2 \mathrm{~h}$ & $\underline{6}$ & 66 \\
$\mathrm{BnNH}_{2}$ & $\mathrm{NaH}$ & $80^{\circ} \mathrm{C}, 3 \mathrm{~h}$ & $\underline{\mathbf{7}}$ & 58 \\
$\mathrm{RM}^{\mathrm{a}}$ & & & complex mixture \\
\hline
\end{tabular}

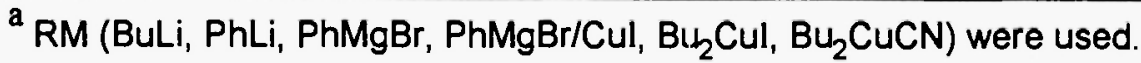




\section{Experimental}

Melting points were determined with a Yanagimoto micro melting point apparatus and are uncorrected. IR spectra were recorded on a JASCO FT/IR 5300 spectrometer. ${ }^{1} \mathrm{H}$ and ${ }^{13} \mathrm{C}$ NMR spectra were obtained with a Varian GEMINI-200 spectrometer at 200 and $50 \mathrm{MHz}$, respectively, for samples in $\mathrm{CDCl}_{3}$ solution with $\mathrm{Me}_{4} \mathrm{Si}$ as an internal standard. ${ }^{19} \mathrm{~F}$ NMR spectra were obtained with a Hitachi FT-NMR R-90F spectrometer at $85 \mathrm{MHz}$ for samples in $\mathrm{CDCl}_{3}$ solutions with $\mathrm{CFCl}_{3}$ as an internal standard. $\mathrm{CI}$ mass spectra were recorded on a JEOL JMS-AX $505 \mathrm{HA}$ mass spectrometer at $70 \mathrm{eV}$ with isobutane as the reagent gas. Flash chromatography was performed with a silica gel column (Fuji-Davison BW-300). Analytical thin-layer chromatography (TLC) was performed on Merck Kieselgel $60 \mathrm{~F}_{254}$. Microanalyses were performed with a Perkin-Elmer $2400 \mathrm{~S}$ CHN elemental analyzer.

(E)-1-(3,3,3-Trifluoro-1-propenylsulfonyl)pyrrolidine 1. To a solution of pyrrolidine $(8.4 \mathrm{~mL}, 100$ $\mathrm{mmol})$ and triethylamine $(10 \mathrm{~mL}, 120 \mathrm{mmol})$ in dry $\mathrm{CH}_{2} \mathrm{Cl}_{2}(100 \mathrm{~mL})$ at $0{ }^{\circ} \mathrm{C}$ was added methanesulfonyl chloride $(9.3 \mathrm{~mL}, 120 \mathrm{mmol})$ under nitrogen atmosphere. After being stirred for $1 \mathrm{~h} \mathrm{at} 0{ }^{\circ} \mathrm{C}$, the solution was poured into $1 \mathrm{M}$ aqueous $\mathrm{K}_{3} \mathrm{PO}_{4}$ solution $(200 \mathrm{~mL})$ and extracted with $\mathrm{CHCl}_{3}(3 \times 100 \mathrm{~mL})$. The combined extracts were washed with $1 \mathrm{M}$ aqueous $\mathrm{HCl}(3 \times 100 \mathrm{~mL})$. The organic layer was dried $\left(\mathrm{MgSO}_{4}\right)$ and evaporated under reduced pressure. The residue was recrystallized from hexane-AcOEt to give $\underline{2}$ as a colorless solid $(14 \mathrm{~g}, 95 \%$ yield), mp $66-69^{\circ} \mathrm{C}$ (lit. (6) $\left.70-71{ }^{\circ} \mathrm{C}\right)$. To a solution of $\underline{2}(14 \mathrm{~g})$ in dry THF $(90 \mathrm{~mL})$ at $-78{ }^{\circ} \mathrm{C}, n$-BuLi $(1.6 \mathrm{M}$ hexane solution, $88 \mathrm{~mL}, 143 \mathrm{mmol}$ ) was added under nitrogen atmosphere in $15 \mathrm{~min}$. The solution was stirred for $15 \mathrm{~min}$ at $-78{ }^{\circ} \mathrm{C}$ and then for additional $1 \mathrm{~h}$ at $0{ }^{\circ} \mathrm{C}$. To the resulting solution was added ethyl trifluoroacetate $\left(28 \mathrm{~mL}, 238 \mathrm{mmol}\right.$ ) in $15 \mathrm{~min}$ at $-78^{\circ} \mathrm{C}$. After being stirred overnight at room temperature, the solution was poured into saturated aqueous $\mathrm{NaCl}$ solution $(150 \mathrm{~mL})$ and extracted with $\mathrm{AcOEt}(3 \times 80 \mathrm{~mL})$. The combined extracts were dried $\left(\mathrm{MgSO}_{4}\right)$ and evaporated under reduced pressure to give the ketone 3 ( $25 \mathrm{~g} ; R_{f} 0.33$ (AcOEt); IR $\left.=3567,1593 \mathrm{~cm}^{-1}\right)$. Without purification, $\mathrm{NaBH}_{4}(5.4 \mathrm{~g}, 143 \mathrm{mmol}$ ) was added to a solution of $\underline{3}$ $(25 \mathrm{~g})$ in $\mathrm{MeOH}(20 \mathrm{~mL})$. After being stirred overnight at room temperature, the solution was poured into saturated aqueous $\mathrm{NaCl}$ solution $(50 \mathrm{~mL})$ and extracted with AcOEt $(3 \times 20 \mathrm{~mL})$. The combined extracts were dried $\left(\mathrm{MgSO}_{4}\right)$ and evaporated under reduced pressure to give the alcohol $4\left(16 \mathrm{~g} ; R_{f} 0.17\left(\mathrm{CH}_{2} \mathrm{Cl}_{2}\right) ; \mathrm{IR}=3430\right.$ $\left.\mathrm{cm}^{-1}\right)$. Without purification, methanesulfonyl chloride ( $\left.7.6 \mathrm{~mL}, 98 \mathrm{mmol}\right)$ was added to a solution of $4(16 \mathrm{~g})$ and triethylamine $(27 \mathrm{~mL}, 197 \mathrm{mmol})$ in dry $\mathrm{CH}_{2} \mathrm{Cl}_{2}(50 \mathrm{~mL})$ at $0{ }^{\circ} \mathrm{C}$ under nitrogen atmosphere. After being stirred for $1 \mathrm{~h}$ at $0{ }^{\circ} \mathrm{C}$, the solution was poured into $1 \mathrm{M}$ aqueous $\mathrm{K}_{3} \mathrm{PO}_{4}$ solution (100 mL) and extracted with $\mathrm{CHCl}_{3}$ $(50 \mathrm{~mL} \times 3)$. The combined extracts were washed with $1 \mathrm{M}$ aqueous $\mathrm{HCl}(3 \times 50 \mathrm{~mL})$. The organic layer was dried $\left(\mathrm{MgSO}_{4}\right)$ and evaporated under reduced pressure. The residue was recrystallized from hexane- $\mathrm{CHCl}_{3}$ to give 1 as a colorless solid $\left(12 \mathrm{~g}, 56 \%\right.$ yield from 2 ): $\mathrm{mp} 45-48{ }^{\circ} \mathrm{C}$; IR (KBr) $1134,1354,1462 \mathrm{~cm}^{-1}$; ${ }^{1} \mathrm{H}$ NMR $\delta$ $1.92-1.99(4 \mathrm{H}, \mathrm{m}), 3.31-3.38(4 \mathrm{H}, \mathrm{m}), 6.69(1 \mathrm{H}, \mathrm{dq}, J=15.2,5.9 \mathrm{~Hz}), 6.88(1 \mathrm{H}, \mathrm{dq}, J=15.2,1.5 \mathrm{~Hz}) ;{ }^{13} \mathrm{C}$ NMR $\delta 25.9,48.1,121.8(\mathrm{q}, J=271 \mathrm{~Hz}), 129.3(\mathrm{q}, J=36 \mathrm{~Hz}), 133.6(\mathrm{q}, J=6 \mathrm{~Hz}) ;{ }^{19} \mathrm{~F}$ NMR $\delta-65.2(\mathrm{~d}, J=6$ $\mathrm{Hz}$ ); MS (CI) $m / 2230\left(\mathrm{M}+\mathrm{H}^{+}\right)$. Anal. Calcd for $\mathrm{C}_{7} \mathrm{H}_{10} \mathrm{~F}_{3} \mathrm{NO}_{2} \mathrm{~S}: \mathrm{C}, 36.68 ; \mathrm{H}, 4.40 ; \mathrm{N}, 6.11$. Found: $\mathrm{C}, 36.62$; $\mathrm{H}, 4.52 ; \mathrm{N}, 5.89$.

1-[3,3-Bis(carbomethoxy)-2-(trifuoromethyl)propylsulfonyl]pyrrolidine $\underline{5}$. To a solution of dimethyl malonate $(159 \mathrm{mg}, 1.2 \mathrm{mmol})$ in $\mathrm{dry}$ THF $(3 \mathrm{~mL})$ at $0{ }^{\circ} \mathrm{C}$ was added $\mathrm{NaH}(60 \%$ oil dispersion, $48 \mathrm{mg}$, $1.2 \mathrm{mmol}$ ) portionwise under nitrogen atmosphere. The solution was stirred for $10 \mathrm{~min}$ at room temperature and then a solution of $1(230 \mathrm{mg}, 1.0 \mathrm{mmol})$ in dry THF $(2 \mathrm{~mL})$ was added. After being stirred for $1 \mathrm{~h}$ at room 
temperature, the solution was poured into saturated aqueous $\mathrm{NaCl}$ solution $(15 \mathrm{~mL})$ and extracted with $\mathrm{CHCl}_{3}(3$ $\times 5 \mathrm{~mL})$. The combined extracts were dried $\left(\mathrm{MgSO}_{4}\right)$ and evaporated under reduced pressure. The residue was chromatographed on a silica gel column ( $3: 1$ hexane : AcOEt) to give $\underline{\mathbf{5}}$ as a yellow solid ( $360 \mathrm{mg}, 100 \%$ yield): mp 42-44 ${ }^{\circ} \mathrm{C}$; IR (KBr) 1746, 1339, $1154 \mathrm{~cm}^{-1}$; ${ }^{1} \mathrm{H}$ NMR $\delta$ 1.92-1.99 (4 H, m), 3.35-3.42 (4 H, m), 3.62-3.76 (3 $\mathrm{H}, \mathrm{m}), 3.79(3 \mathrm{H}, \mathrm{s}), 3.81(3 \mathrm{H}, \mathrm{s}), 4.02(1 \mathrm{H}, \mathrm{d}, J=3.8 \mathrm{~Hz}) ;{ }^{13} \mathrm{C} \mathrm{NMR} \delta 25.9,39.8(\mathrm{q}, J=29 \mathrm{~Hz}), 44.8(\mathrm{q}, J=2$ $\mathrm{Hz}$ ), 48.0, 48.7, 53.2, 53.5, 125.9 (q, $J=280 \mathrm{~Hz}), 167.2,167.5 ;{ }^{19} \mathrm{~F}$ NMR $\delta-69.4$ (d, $\left.J=7 \mathrm{~Hz}\right)$; MS (CI) $m / s$ $362\left(\mathrm{M}+\mathrm{H}^{+}\right)$. Anal. Calcd for $\mathrm{C}_{12} \mathrm{H}_{18} \mathrm{~F}_{3} \mathrm{NO}_{6} \mathrm{~S}: \mathrm{C}, 39.89 ; \mathrm{H}, 5.02 ; \mathrm{N}, 3.88$. Found: $\mathrm{C}, 39.94 ; \mathrm{H}, 5.00 ; \mathrm{N}, 3.83$.

1-[4-Oxo-4-phenyl-2-(trifluoromethyl)propylsulfonyl]pyrrolidine $\underline{6}$. To a solution of LDA, prepared from $n$-BuLi (1.6 M hexane solution, $1.4 \mathrm{~mL}, 2.2 \mathrm{mmol}$ ) and diisopropylamine (202 $\mathrm{mg}, 2.0 \mathrm{mmol})$ in dry THF $(3 \mathrm{~mL})$ at $-78^{\circ} \mathrm{C}$, acetophenone $(180 \mathrm{mg}, 1.5 \mathrm{mmol})$ was added under nitrogen atmosphere at $-78^{\circ} \mathrm{C}$ in $5 \mathrm{~min}$. The solution was stirred for $1 \mathrm{~h}$ at $-78^{\circ} \mathrm{C}$ and then a solution of $1(230 \mathrm{mg}, 1.0 \mathrm{mmol})$ in THF ( $\left.2 \mathrm{~mL}\right)$ was added. After being stirred for $1 \mathrm{~h}$ at $-78^{\circ} \mathrm{C}$, the solution was poured into saturated aqueous $\mathrm{NaCl}$ solution (15 $\mathrm{mL})$ and extracted with AcOEt $(3 \times 10 \mathrm{~mL})$. The combined extracts were dried $\left(\mathrm{MgSO}_{4}\right)$ and evaporated under reduced pressure. The residue was chromatographed on a silica gel column ( $2: 1$ hexane : AcOEt) to give $\underline{6}$ as a

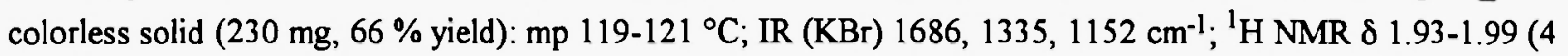
$\mathrm{H}, \mathrm{m}), 3.19(1 \mathrm{H}, \mathrm{dd}, J=14.4,9.8 \mathrm{~Hz}), 3.30-3.70(7 \mathrm{H}, \mathrm{m}), 3.72-3.84(1 \mathrm{H}, \mathrm{m}), 7.44-8.00(5 \mathrm{H}, \mathrm{m}) ;{ }^{13} \mathrm{C}$ NMR $\delta$ $26.0,35.1$ (q, $J=28 \mathrm{~Hz}), 36.3(\mathrm{q}, J=2 \mathrm{~Hz}), 47.4$ (q, $J=2 \mathrm{~Hz}), 48.0,127.0$ (q, $J=279 \mathrm{~Hz}), 128.5,129.1,134.0$, 136.5, 195.8; ${ }^{19} \mathrm{~F}$ NMR $\delta-65.2(\mathrm{~d}, J-10 \mathrm{~Hz})$; MS (CI) $m / z 350\left(\mathrm{M}+\mathrm{H}^{+}\right)$. Anal. Calcd for $\mathrm{C}_{15} \mathrm{H}_{18} \mathrm{~F}_{3} \mathrm{NO}_{3} \mathrm{~S}: \mathrm{C}$, 51.57 ; H, 5.19; N, 4.01. Found: C, 51.40; H,5.10; N, 4.01 .

1-[2-(Benzylamino)-3,3,3-trifluoropropylsulfonyl]pyrrolidine 7 . To a solution of 1 (230 $\mathrm{mg}, 1.0$ $\mathrm{mmol})$ and benzylamine $(118 \mathrm{mg}, 1.1 \mathrm{mmol})$ in THF $(3 \mathrm{~mL})$ at $0{ }^{\circ} \mathrm{C}$ was added $\mathrm{NaH}(60 \%$ oil dispersion, $60 \mathrm{mg}$, $1.5 \mathrm{mmol}$ ) under nitrogen atmosphere. After being stirred for $3 \mathrm{~h}$ at $80^{\circ} \mathrm{C}$, the solution was poured into saturated aqueous $\mathrm{NaCl}$ solution $(20 \mathrm{~mL})$ and extracted with AcOEt $(3 \times 10 \mathrm{~mL})$. The combined extracts were dried $\left(\mathrm{MgSO}_{4}\right)$ and evaporated under reduced pressure. The residue was chromatographed on a silica gel column $(2: 1$ hexane : AcOEt) to give $\underline{7}$ as a yellow oil $\left(96 \mathrm{mg}, 58 \%\right.$ yield): $R_{f} 0.46(2: 1$ hexane : AcOEt); IR (neat) 1263 , $1152,1127 \mathrm{~cm}^{-1} ;{ }^{1} \mathrm{H}$ NMR $\delta 1.79-1.98(4 \mathrm{H}, \mathrm{m}), 2.16(1 \mathrm{H}, \mathrm{br} \mathrm{s}), 3.06(1 \mathrm{H}, \mathrm{dd}, J=14.2,9.8 \mathrm{~Hz}), 3.24(1 \mathrm{H}$, dd, $J=14.2,2.4 \mathrm{~Hz}), 3.29-3.42(4 \mathrm{H}, \mathrm{m}), 3.75(1 \mathrm{H}, \mathrm{dqd}, J=9.8,6.8,2.4 \mathrm{~Hz}), 3.92(1 \mathrm{H}, \mathrm{d}, J=12.6 \mathrm{~Hz}), 4.03(1 \mathrm{H}$, $\mathrm{d}, J=12.6 \mathrm{~Hz}$ ), 7.24-7.40 (5 H, m); ${ }^{13} \mathrm{C}$ NMR $\delta 25.9,47.8,47.9,53.0,56.0$ (q, $\left.J=29 \mathrm{~Hz}\right), 126.0(\mathrm{q}, J=283$ $\mathrm{Hz}), 127.8,128.9,128.9,139.1 ;{ }^{19} \mathrm{~F} \mathrm{NMR} \delta-75.5(\mathrm{~d}, J=7 \mathrm{~Hz})$; MS (CI) $m / 2337\left(\mathrm{M}+\mathrm{H}^{+}\right)$. Anal. Calcd for $\mathrm{C}_{14} \mathrm{H}_{19} \mathrm{~F}_{3} \mathrm{~N}_{2} \mathrm{O}_{2} \mathrm{~S}: \mathrm{C}, 49.98 ; \mathrm{H}, 5.69 ; \mathrm{N}, 8.33$. Found: $\mathrm{C}, 50.08 ; \mathrm{H}, 5.98 ; \mathrm{N}, 7.98$.

\section{References}

(1) (a) G. Resuati, Tetrahedron $\underline{49}, 9385$ (1993); (b) J. T. Welch, S. Eswarakrishnan, Fluorine in Bioorganic Chemistry, John Wiley, New York, 1991; (c) J. T. Welch, Tetrahedron 43, 3123 (1987).

(2) K. Uneyama, J. Synth. Org. Chem., Jpn. 49, 612 (1991), and references cited therein.

(3) H. Tsuge, T. Okano and S. Eguchi, J. Chem. Soc., Perkin Trans. 12761 (1995).

(4) (a) Y. Kawano, T. Watanabe, J. Sakai, H. Watanabe, M. Nagano, T. Nishimura and T. Miyadera, Chem. Pharm. Bull. 28, 70 (1980); (b) D. V. Santi and T. T. Sakai, Biochemistry 10, 3598 (1971).

(5) P. Perlmutter, Conjugate Addition Reactions in Organic Synthesis, Pergamon Press, Oxford, 1992.

(6) J. Sacco, Jr, P. Z. Anthony, D. R. Borgen and L. G. Ginger, J. Am. Chem. Soc. $\underline{76}, 303$ (1954).

Received on Septenber 20, 1886 